\title{
A physical interpretation of the use of fractional operators for modelling the drying process
}

\author{
Perré, P. ${ }^{\mathrm{a}, \mathrm{b}}$; Turner, $\mathbf{I}^{\mathrm{c}}$ \\ a LGPM, CentraleSupélec, Université Paris-Saclay, 91190 Gif-sur-Yvette, France \\ b LGPM, CentraleSupélec, Centre Européen de Biotechnologie et de Bioéconomie (CEBB), 51110 \\ Pomacle, France. \\ 'School of Mathematical Sciences and ARC Centre of Excellence for Mathematical and Statistical \\ Frontiers, Queensland University of Technology, Brisbane, Australia
}

*E-mail of the corresponding author: patrick.perre@centralesupelec.fr

\begin{abstract}
Fractional order derivatives provide useful alternatives to their integer order counterparts due to their ability to model memory and other properties of the porous medium, such as nonlocal behaviour. These phenomena are driven by the constrained interactions within the complex and non-homogeneous microstructures evident at the pore scale.

In this work, we investigate the suitability of time and space fractional operators for modelling drying processes and provide a physical interpretation of these operators. At first, the concept and the general formulation in the case of a 1-D finite domain is summarised. Then a selection of simulations allowed us to analyse the physical effects of these operators on the solution. In particular, we elucidate:(I the ability of these operators to break the fundamental relationship between mean square displacement and time in the simple example of diffusion in an open space, (ii) the caution to be taken with the formulation of boundary conditions and source terms to obtain consistent balance equations, (iii) the effect of fractional in space diffusion as a way to alter the MC profiles compared to standard diffusion, therefore potentially avoiding the dependence of the diffusivity on the variable
\end{abstract}

Keywords: Fractional Calculus; Transport in Porous Media; Finite Volume Method; Matrix Transfer Technique; Matrix Functions. 


\section{Introduction}

Modelling the transport of moisture through heterogeneous porous media finds application across a number of important environmental problems, such as drying [1] and groundwater flow in freshwater coastal aquifers [2-4]. Experimental work performed by Perré et al. [5] highlighted that the traditional diffusion equation did not adequately describe the absorption of water in the cell wall of beech wood. An unrealistically small diffusion coefficient was required to best fit the experimental data, which was clearly impractical. The observed experimental phenomenon exhibited a large deviation from the stochastic process of Brownian motion, which is a typical case of anomalous, or non-Fickian, diffusion. The bound water migration through the cell walls was thought to be anomalous because the water molecules that are bound to the macromolecules via their hydrogen bonds move by successive jumps within the complex structure of these macromolecules. This finding motivated us to explore alternate modelling approaches for this process.

Based on this assumption, Turner et al. [1] derived a coupled anomalous transport model, which introduced a moisture potential linked to the moisture content field via a non-local fractional-in-space operator involving the Laplacian raised to a negative fractional index. The simulation results highlighted that this model accurately described the absorption of water in the cell wall and provided a good overall mass balance error. Most importantly however, good agreement with experimental data for a suitable range of diffusivity values was achieved only for a fractional index of $\alpha=0.76$ ( $\alpha=2$ is standard Fickian diffusion).

Both Turner et al. [1] and Fomin et al. [6,7] proposed a fractional-in-space model of the form:

$$
\frac{\partial X}{\partial t}=-D_{a} D_{b}(-\Delta)^{\frac{a}{2}} X
$$

for the so-called super-diffusion of moisture in the porous matrix. In Fomin et al. [6,7] the Caputo definition is adopted for the fractional-in-space operator, whereas in Turner et al. [1] the fractional Laplacian is defined in terms of its spectral decomposition together with homogeneous boundary conditions (BCs). One notes, however, that it can be shown using Laplace transforms that the two definitions are equivalent on semi-infinite domains.

The solution of (1) with standard (Dirichlet, Neumann or Robin) inhomogeneous BCs has been explored previously by the authors [8-10]. In deriving equation (1) the following expression for the moisture flux was used:

$$
j=-\rho_{0} D_{b} \nabla \mathcal{X}
$$

where $c$ was defined as the moisture potential. 
Using this expression for the moisture flux leads to the BCs at the exposed surface $\partial \Omega$ of the domain $\Omega$ being defined for a given function $\boldsymbol{f}$ as

$$
\boldsymbol{j} \cdot \boldsymbol{n}=f(X) \text { on } \partial \Omega
$$

Clearly BC (3) is nonlinear and very challenging even for the standard problem $\alpha=2$. One of the aims of this research is to provide an analysis of such problems. Another important contribution of this work is the extension of the model (1-3) to include both fractional in time and space operators in the mass conservation balance law, as well as a nonlinear source term $g(u)$. The derivation of analytical and numerical solutions is quite complex and will therefore not be presented here. We focus primarily on the following:

- The best fractional modelling framework to ensure mass conservation is achieved.

- Whether fractional in space diffusion can be used as a way to avoid the dependence of the diffusivity on the variable.

- The effect of the material thickness on the global behaviour for fractional in space or fractional in time formulations.

We also pay careful attention to the caution that must be taken with the formulation of boundary conditions and source terms to obtain consistent balance equations.

\section{Fractional Transport Model}

Before commencing the model derivation, we provide some preliminary background information on the various definitions of the fractional in time and space operators used throughout the paper.

\section{Definition 1. Caputo fractional derivatives on a finite interval [11]}

Let $[\boldsymbol{a}, \boldsymbol{b}]$ be a finite interval of the real axis, $\alpha>0, n-1<\alpha<n, n \in \mathbb{N}$, then the left sided Caputo fractional derivatives with order $\alpha$ is given by

$$
{ }^{C} D_{a-}^{\alpha} f(t)=\frac{1}{\Gamma(n-\alpha)} \int_{a}^{t} \frac{f^{(f)}(\xi) d \xi}{(t-\xi)^{\alpha-n+1}}
$$

where the gamma function is defined as $\Gamma(t)=\int_{0}^{\infty} t^{z-1} e^{-t} d t, \Re(z)>0$ with $t^{z-1}=e^{(z-1) \log t}$ and $\log t \in \mathbb{R}$.

\section{Definition 2. Riemann-Liouville fractional derivatives [11]}

Let $[a, b](-\infty<a<b<\infty)$ be a finite interval of the real axis, $f(l) \in A C^{n-1}([a, b])$ and $n-1<\alpha<n, n \in \mathbb{N}$, then the left-sided Riemann-Liouville fractional derivative of order $\alpha$ is given as 


$$
{ }^{R L} D_{a+f}^{\alpha} f(t)=\frac{1}{\Gamma(n-\alpha)} \frac{d^{n}}{d t^{n}} \int_{a}^{t} \frac{f(\xi) d \xi}{(t-\xi)^{\alpha-n+1}}
$$

\section{Definition 3. (Fractional Laplacian) $[8,9,10]$}

Let $\left\{\varphi_{n}\right\}$ be a complete set of orthonormal eigenfunctions corresponding to eigenvalues $\lambda_{n}^{2}$ of the operator $\left(-\frac{d^{2}}{d x^{2}}\right)$ on a bounded domain $-L<x<L$, with homogeneous boundary conditions $\mathcal{B}(\varphi)=0$ defined at $\quad x=-L \quad$ and $\quad x=L$. Let $\mathcal{F}_{\gamma}=\left\{f \in C(\Omega)\left|\sum_{n=1}^{\infty}\right| \lambda_{n} \|\left. c_{n}\right|^{2}<\infty, c_{n}=\left\langle f, \varphi_{n}\right\rangle, \gamma=\max (2 \alpha, 0)\right\}$. Then, for any $f \in \mathcal{F}_{\gamma}$ we define $\left(-\frac{\partial^{2}}{\partial x^{2}}\right)^{\alpha / 2} f=\sum_{n=1}^{\infty} \lambda_{n}^{\alpha} c_{n} \varphi_{n}$.

\subsection{Fractional Modelling Framework}

Assuming the solid matrix is rigidly fixed, we can express the mass $m=\rho_{0} X$ where $\boldsymbol{X}$ is the moisture content. The flux of mass is taken as either standard (Fickian) diffusion ( $\alpha=2$ ) with $\boldsymbol{j}=-\rho_{0} D_{b} \nabla X$, or anomalous (non-Fickian) diffusion $(0<\alpha<2)$ with $\bar{j}=-\rho_{0} D_{b} \nabla \mathcal{X}$ and the moisture potential defined as $\mathcal{X}=D_{a}(-\Delta)^{2} X$. The constant $D_{b}$ has the usual dimensions of diffusivity $\left(m^{2} s^{-1}\right)$, whereas the constant $D_{a}$ is introduced, if for no other reason, to recover the correct units for the flux, i.e. $D_{a}=1 \mathrm{~m}^{\alpha-2}$. To complete the formulation, one needs an initial condition and boundary conditions, which are usually given in terms of a mass flux that is typically a nonlinear function of $\boldsymbol{X}$. A onedimensional framework for these fractional models provides the perfect mechanism by which to test these important hypotheses and to study the evolution of the solution behaviour. In order to achieve these objectives, we discuss the following generalised onedimensional model that must be solved for $X$ :

\subsection{General formulation - Fractional in Time and Space:}

$$
\begin{gathered}
\frac{\partial X}{\partial t}={ }^{R L} D_{0+}^{1-\gamma}\left(D_{b} \frac{\partial^{2} \mathcal{X}}{\partial x^{2}}\right)+g(X),-L<x<L, t>0,0<\alpha \leq 2 \\
{ }^{R L} D_{0 \mid}^{1-\gamma}\left(-D_{b} \frac{\partial \mathcal{X}}{\partial x}(-L, t)+\sigma_{L} \mathcal{X}\right)=f_{1}(X), x=-L, t>0 \\
{ }^{R L} D_{0+}^{1}\left(D_{b} \frac{\partial \mathcal{X}}{\partial x}(L, t)+\sigma_{R} \mathcal{X}\right)=f_{2}(X), x=L, t>0 \\
X(x, 0)=X_{0}(x) \text {, with } 0<\gamma<1,0<\alpha \leq 2
\end{gathered}
$$


In this formulation, $\sigma_{L}, \sigma_{R}$ are regularisation parameters with the choice $\sigma=0$ giving Neumann boundary conditions (suitable when only time fractional operators are employed); $\sigma \rightarrow \infty$ gives Dirichlet conditions and otherwise mixed or Robyn type conditions prevail. We explore finite values of $\sigma(\sigma \ll 1)$ to ensure non-singularity of the fractional potential operator $(-\Delta)^{\alpha / 2-1}$ at the smallest eigenvalue. The functions $f_{1}(X), f_{2}(X), g(X)$ and $\boldsymbol{X}_{\mathbf{0}}(\boldsymbol{x})$ are assumed smooth and defined in the context of the problem. We also use the relationship between the Caputo and Riemann-Liouville fractional derivatives given in [11].

A key point in our investigations will be the way in which the fractional operators are applied to the boundary fluxes and source terms to ensure consistent physical behaviour is observed and that an overall mass conservation is achieved.
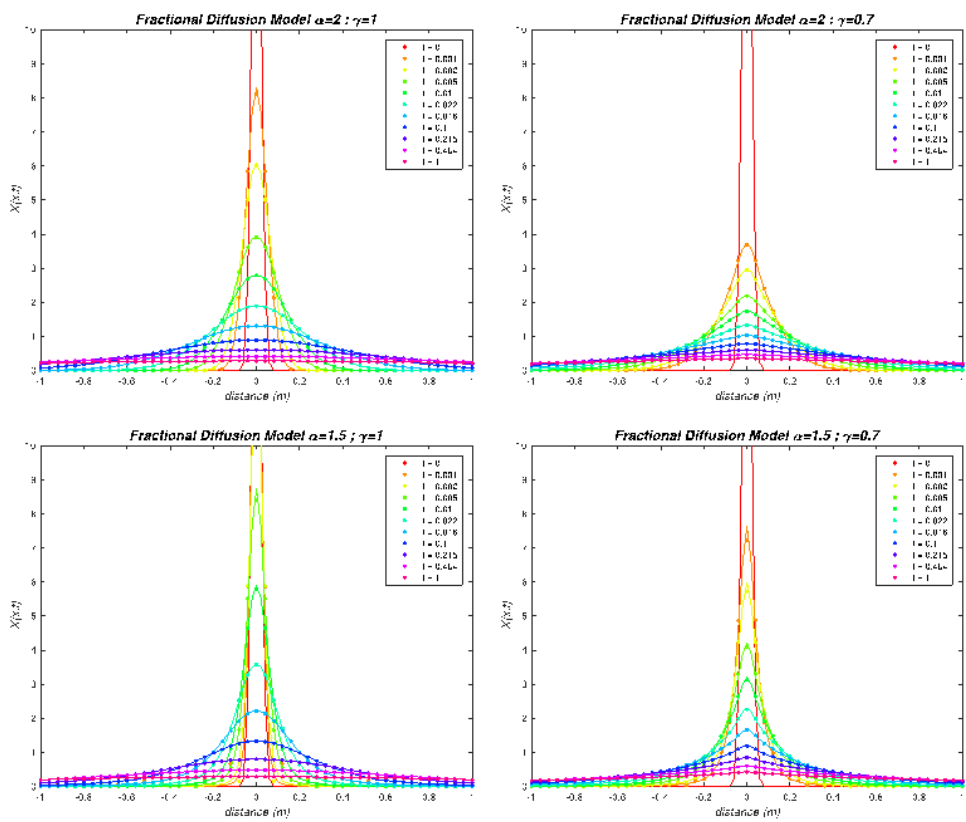

Fig. 1 Plots of moisture content profiles for test problem 1 at various times for (a) standard diffusion ( $\alpha=2, \gamma=1)$; (b) fractional in time ( $\alpha=2, \gamma=0.7)$; (c) fractional in space $(\alpha=1.5, \gamma=1)$; (d) Fractional in time and space $(\alpha=1.5, \gamma=0.7)$.

\section{Results and discussion}

The first problem presented here concerns the time-evolution of an initial field in an infinite domain. Therefore, neither BCs, nor source terms are involved in the model formulation. To come back to the basic definition of diffusion, the initial profile is chosen as a narrow Gaussian curve, likely to be close to the diffusion of a Dirac function (Fig. 1). Consistently, 
the mean square displacement (MSD) is proportional to time for normal diffusion (Fig. 2a). This slope equals the fractional value $\gamma$ for the fractional in time diffusion (Fig. 2b).

In this case, the total mass is conserved, however the allocation of this mass over time is very different from standard diffusion: the center value decreases rapidly at short times but is much slower at longer times compared to normal diffusion. The fractional in space diffusion produces a MSD quasi-proportional to time, which is similar to normal diffusion. However, this MSD is obtained with different spatial fields: the center peak is higher than for normal diffusion at similar times, but this is compensated by a slight moisture increase over long distances, even at short times. The fractional in time and space diffusion accumulates these trends (Fig. 1d).
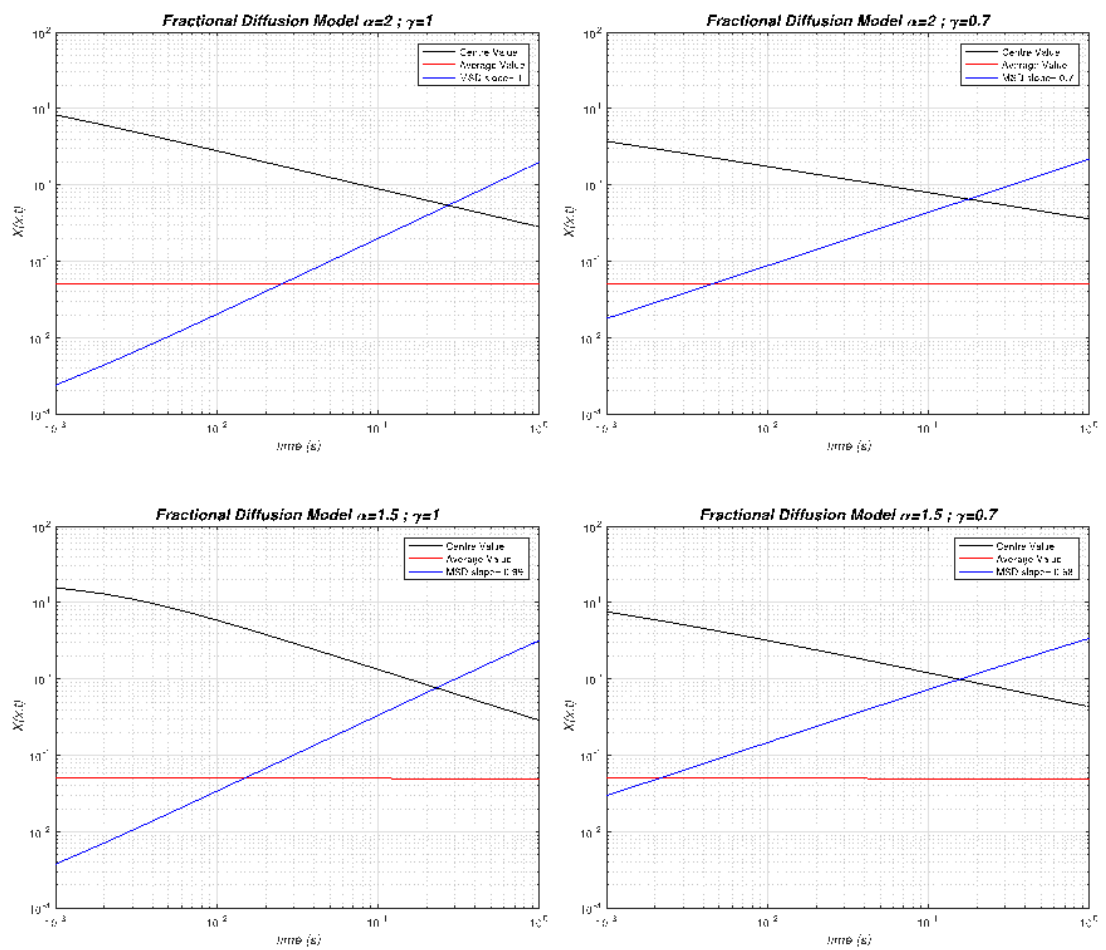

Fig. 2 Plots of means square displacement (MSD), average and centre value for test problem 1 (a) standard diffusion; (b) fractional in time ( $\alpha=2, \gamma=0.7)$; (c) fractional in space $(\alpha=1.5, \gamma=1)$; (d) Fractional in time and space $(\alpha=1.5, \gamma=0.7)$.

The solution of the general problem when BCs and/or source terms are involved is more challenging. In particular, great care should be taken in the way the fractional operator has to be applied to the source term and boundary fluxes for the mass balance to be respected. Due to space restrictions, these developments cannot be presented here. 
As a final example, we show in Fig. 3 simulation results for different assumptions in the absence of a source term and with Newman boundary conditions (obtained in the general formulation with $\sigma=0.001$ ). The standard diffusion exhibits a classical behavior as a result of the flux imposed at the boundaries. The profiles first establish and maintain the same shape which is just regularly shifted towards lower values along time to respect the mass balance. In Fig. 3a, the distance between two successive profiles is exactly the same, as the profiles are plotted with constant time intervals. At first sight, the fractional in time solution (Fig. 3b) is similar to standard diffusion. Note however that the profiles evolve in shape, becoming steeper and steeper as time advances. This effect is more spectacular for the fractional in space solution (Fig. 3c). In this case, the profiles are very steep near the exchange faces. In this case, the spatial rather than the temporal effects modify the local ability of the flux to establish as a function of the moisture gradient.
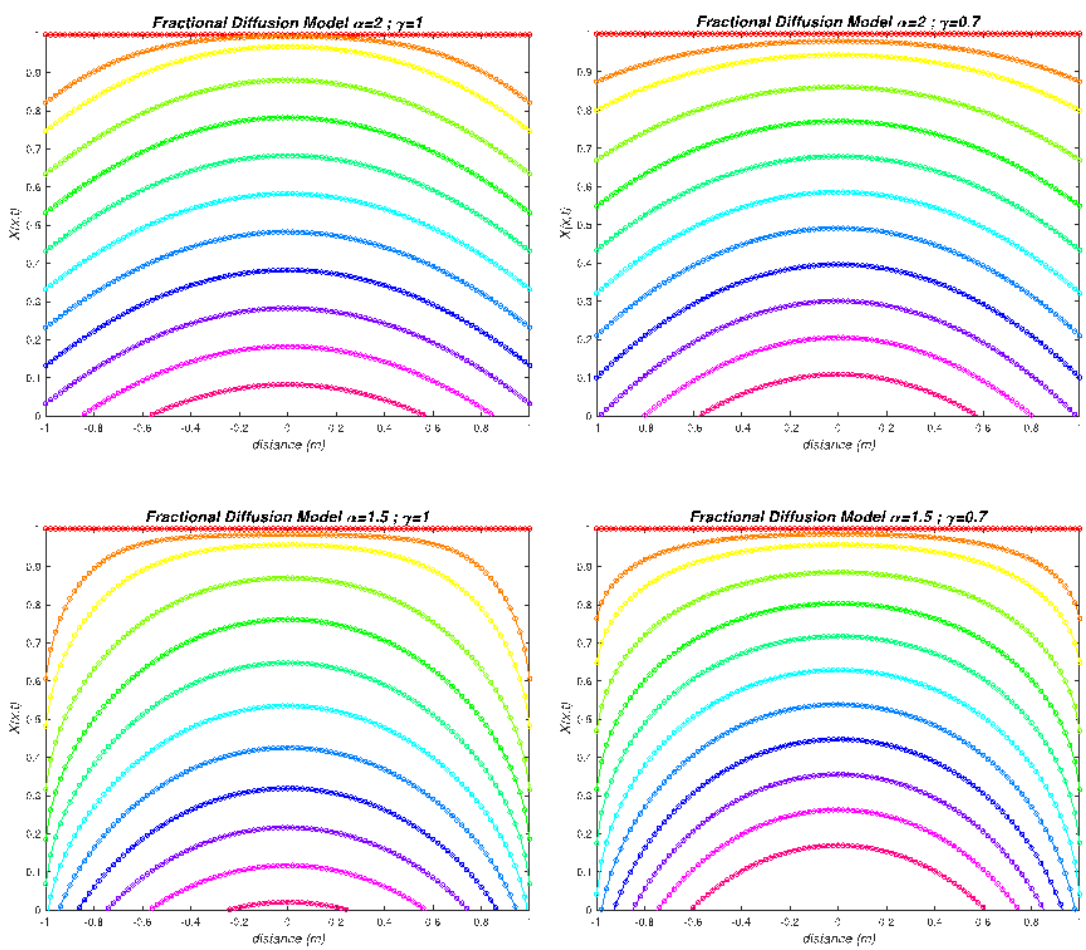

Fig. 3 Plots of moisture content profiles for Newman BC at various times for (a) standard diffusion; (b) fractional in time ( $\alpha=2 . \gamma=0.7)$; (c) fractional in space ( $\alpha=1.5, \gamma=1)$; (d) Fractional in time and space $(\alpha=1.5, \gamma=0.7)$.

The fractional in time and in space solution (Fig. 3d) combines these two effects: again, the fractional in time operator acts more particularly at longer times, for which the profiles are steep both at the boundaries due the fractional in space operator and inside the slab due to 
the fractional in time operator. One can easily imagine that when combined, these two behaviors may address different kinds of experimental observation.

\section{Conclusion}

In this work, we investigated the suitability of fractional operators for simulating drying processes and we also provided a physical interpretation of these operators. At first, a general formulation in the case of a 1-D finite domain was summarised. Then a selection of simulations allowed us to identify the physical effects of these operators on the solution behaviour. In particular, we highlighted:

- The ability of these operators to break the fundamental relationship between mean square displacement and time in the simple example of diffusion in an open space,

- The caution to be taken with the formulation of boundary conditions and source terms to obtain consistent balance equations (not detailed here because of the space restrictions),

- The effect of space fractional diffusion to change the MC profiles compared to standard diffusion, therefore potentially avoiding the dependence of the diffusivity on the variable.

\section{Acknowledgements}

This work was supported financially by a visiting Professorial Fellowship that enabled Turner to work at CentraleSupélec, Université Paris-Saclay, France for a period of three months. Both authors acknowledge the financial support for this research received through the Australian research Council (ARC) grant DP150103675.

\section{References}

[1] I. Turner, M. Ilic and P. Perré, Drying Technology, 2011, 29, 1932 - 1940.

[2] R. Chittaranjan, T.R. Ellsworth, A.J. Valocchi and C.W. Boast, J. Hydrology, 1997, 193, 270-292.

[3] Y. Zhang, D.A. Benson, D.M. Reeves, Advances in Water Resources, 2009, 32:561581.

[4] D. A. Benson, S.W. Wheatcraft and M.M. Meerschaert, Water Resources Res., 2000, 36, 1413-1423.

[5] P. Perré, A.C. Houngan and P. Jacquin, Drying Technology, 2007, 25:1341-1347.

[6] S. Fomin, V. Chugunov, T. Hashida, Proc. R. Soc. A, 2005, 461, 2923-2939.

[7] S. Fomin, V. Chugunov, T. Hashida, Transp. Porous Med., 2010, 81,187-205.

[8] M. Ilić, F. Liu, I. Turner, and V. Anh, Fract. Calc. Appl. Anal., 2005, 8, 323-341.

[9] M. Ilić, F. Liu, I. Turner, and V. Anh, Fract. Calc. Appl. Anal., 2006, 9, 333-349. istepi

[10] M. Ilić, I. Turner, and V. Anh, Journal of Applied Mathematics and Stochastic

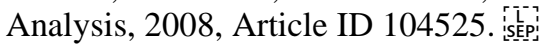

[11] F. Liu, P. Zhuang and Q. Liu, Science Press, China, November 2015, ISBN 978-7-03046335-7. 